\title{
Location-Aware Graph Convolutional Networks for Video Question Answering
}

\author{
Deng Huang, ${ }^{1 *}$ Peihao Chen, ${ }^{1 *}$ Runhao Zeng, ${ }^{1}$ Qing Du, ${ }^{1}$ Mingkui Tan, ${ }^{1,2 \dagger}$ Chuang Gan ${ }^{3}$ \\ ${ }^{1}$ South China University of Technology, ${ }^{2}$ Peng Cheng Laboratory, Shenzhen, ${ }^{3}$ MIT-IBM Watson AI Lab \\ sehuangdeng@mail.scut.edu.cn, \{duqing, mingkuitan\}@ scut.edu.cn, \{phchencs, runhaozeng.cs, ganchuang1990\}@ gmail.com
}

\begin{abstract}
We addressed the challenging task of video question answering, which requires machines to answer questions about videos in a natural language form. Previous state-of-the-art methods attempt to apply spatio-temporal attention mechanism on video frame features without explicitly modeling the location and relations among object interaction occurred in videos. However, the relations between object interaction and their location information are very critical for both action recognition and question reasoning. In this work, we propose to represent the contents in the video as a locationaware graph by incorporating the location information of an object into the graph construction. Here, each node is associated with an object represented by its appearance and location features. Based on the constructed graph, we propose to use graph convolution to infer both the category and temporal locations of an action. As the graph is built on objects, our method is able to focus on the foreground action contents for better video question answering. Lastly, we leverage an attention mechanism to combine the output of graph convolution and encoded question features for final answer reasoning. Extensive experiments demonstrate the effectiveness of the proposed methods. Specifically, our method significantly outperforms state-of-the-art methods on TGIF-QA, Youtube2Text-QA and MSVD-QA datasets.
\end{abstract}

\section{Introduction}

Recently, deep learning has witnessed a great process (Tan, Tsang, and Wang 2014; Cao et al. 2018; 2019; Gan et al. 2019; Guo et al. 2019a). Video question answering (video QA) has become an emerging task in computer vision and has drawn increasing interests over the past few years due to its vast potential applications in artificial question answering system and robot dialogue, video retrieval, etc. In this task, a robot is required to answer a question after watching a video. Unlike the well-studied Image Question Answering (image QA) task which focuses on understanding static images (Anderson et al. 2018; Singh et al. 2018;

\footnotetext{
${ }^{*}$ Equal contribution.

${ }^{\dagger}$ Corresponding author.

Copyright (C) 2020, Association for the Advancement of Artificial Intelligence (www.aaai.org). All rights reserved.
}

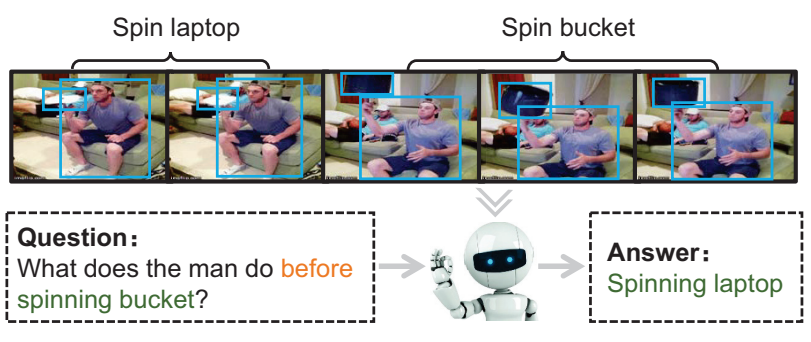

Figure 1: One question-answer (QA) pair in video QA task. To answer the question, the model is required to recognize actions (labeled in green) from the interaction between objects (labeled in blue boxes) and be aware of their temporal order (e.g., before).

Xiong, Merity, and Socher 2016), video QA is more practical since the input visual information often change dynamically, as shown in Figure 1.

Compared with image QA, video QA is much more challenging due to several reasons. (1) Visual content is more complex in a video since it may contain thousands of frames, as shown in Figure 1. More importantly, some frames may be dominated with strong background content which however is irrelevant to questions. (2) Videos often contain multiple actions, but only a part of them are of interest to questions. (3) Questions in video QA task often contain queries related to temporal cues, which implies we should consider both temporal location of objects and complex interaction between them for answer reasoning. For example in Figure 1 , to answer the question "What does the man do before spinning bucket?", the robot should not only recognize the actions "spin laptop" and "spin bucket" by understanding the interaction between the man and objects (i.e., laptop and bucket) in different frames, but also find out the temporal order of actions (e.g., beforelafter) for answer reasoning along time axis.

Taking video frames as inputs, most existing methods (Chenyou Fan 2019; Li et al. 2019b) employ some spatiotemporal attention mechanism on frame features to ask the network "where and when to look". However, these methods are often not robust due to complex background content in 
videos. Lei et al. (Lei et al. 2018) tackle this problem by detecting the objects in each frame and then processing the sequence of object features via an LSTM. However, the order of the input object sequence, which may affect the performance, is difficult to arrange. More importantly, processing the objects in a recurrent manner will inevitably neglect the direct interaction between nonadjacent objects. This is critical for video QA (see experiments in Section 4.4).

In this paper, we introduce a simple yet powerful network named Location-aware Graph Convolutional Networks (LGCN) to model the interaction between objects related to questions. We propose to represent the content in a video as a graph and identify actions through graph convolution. Specifically, the objects of interest are first detected by an off-the-shelf object detector. Then, we construct a fully-connected graph where each node is an object and the edges between nodes represent their relationship. We further incorporate both spatial and temporal object location information into each node, letting the graph be aware of the object locations. When performing graph convolution on the object graph, the objects directly interact with each other by passing message through edges. Last, the output of GCNs and question features are fed into a visualquestion interaction module to predict a answer. Extensive experiments demonstrate the effectiveness of the proposed location-aware graph. We achieve state-of-the-art results on TGIF-QA, Youtube2Text-QA and MSVD-QA datasets.

The main contributions of the proposed method are as follows: (1) we propose to explore actions for video QA task through learning interaction between detected objects such that irrelevant background content can be explicitly excluded; (2) we propose to model the relationships between objects through GCNs such that all objects are able to interact with each other directly; (3) we propose to incorporate object location information into graph such that the network is aware of the location of a specific action; (4) our method achieves state-of-the-art performance on TGIF-QA, Youtube2Text-QA and MSVD-QA datasets.

\section{Related Work}

Visual Question Answering (VQA) is a task to answer the given question based on the input visual information.

Based on the visual sources, we can classify the VQA tasks into two categories: image QA (Goyal et al. 2017; Gan et al. 2017) and video QA (Lei et al. 2018; Yi et al. 2019). Image QA focuses on spatial information. Most image QA models adopt attention mechanism to capture spatial area that related to question words. Yang et al. (Yang et al. 2016) proposed a multi-layer Stacked Attention Network (SAN) which uses questions as query to extract the image region related to the answer. Anderson et al. (Anderson et al. 2018) combined bottom-up and top-down attention which connect questions to specific objects detected by Faster-RCNN. After that, associating feature vector with visual regions becomes a popular framework in the VQA research (i.e. Pythia (Singh et al. 2018)). Xiong et al. (Xiong, Merity, and Socher 2016) introduced the dynamic memory network (DMN) architecture to image QA, which strengthens the reasoning ability of network.
In video QA task, understanding untrimmed videos (Zeng et al. 2019a; Wu et al. 2019) is important. To this end, Jang et al. (Jang et al. 2017) utilized both motion (i.e. C3D) and appearance (i.e., ResNet (He et al. 2016)) features to better represent the video. Li et al. (Li et al. 2019b) replaced RNN with self-attention together with location encoding to model long-range dependencies. However, all the existing methods neglect the interaction between objects, which is vital for video QA task.

Graph-based reasoning has been popular in recent years (Zeng et al. 2019b; Guo et al. 2019b) and shown to be powerful for relation reasoning. To dynamically learn graph structures, CGM (Tan et al. 2015) applied a cutting plane algorithm to iteratively activate a group of cliques. Recently, Graph Convolution Networks (GCNs) (Kipf and Welling 2017) have been used for semi-supervised classification. In text-based tasks, such as machine translation and sequence tagging, GCNs breaks the sequence restriction between each word and learns the graph weight by attention mechanism, which makes it work better in modeling longer sequence than LSTM. Some methods (Norcliffe-Brown, Vafeias, and Parisot 2018; Cadène et al. 2019; Li et al. 2019a) took into consideration the object position for image QA tasks. In video recognition, Wang et al. (Wang and Gupta 2018) proposed to use GCNs to capture relations between objects in videos, where objects are detected by an object detector pretrained on extra training data. Despite their success, there is no efficient graph model for video QA task.

Attention mechanism has been leveraged in various tasks. Several works (Gan et al. 2015; Long et al. 2018) used attention model to improve the performance on video recognition. Vaswani et al. (Vaswani et al. 2017) utilized selfattention mechanism for language translation and (Nguyen and Okatani 2018) proposed Co-Attention which can be stacked to form a hierarchy for multi-step interactions between visual and language features. Jang et al. (Jang et al. 2017) proposed a simple baseline which uses both spatial and temporal attention to reason the video and answer the question. In our proposed method, we use attention mechanism to fuse video and question modalities.

\section{Proposed Method}

\subsection{Notation and Problem Definition}

Given a video containing $N$ frames with $K$ detected objects on each frame, let $\mathcal{R}=\left\{\mathbf{o}_{n, k}, \mathbf{b}_{n, k}\right\}_{n=1, k=1}^{n=N, k=K}$ be the detected object set, where o denotes the object feature obtained by RoIAlign (He et al. 2017) and $\mathbf{b}$ is the spatial location of each object. We use $T=N \times K$ to denote the total number of objects in one video. We denote a graph as $\mathcal{G}=(\mathcal{V}, \mathcal{E})$ with $M$ nodes $\mathbf{v}_{i} \in \mathcal{V}$ and edges $e_{i j} \in \mathcal{E}$. The adjacency matrix of graph is represented as $\mathbf{A} \in \mathbb{R}^{M \times M}$. The question with $\kappa$ words is denoted as $\mathrm{Q}$.

In this paper, we focus on video QA task, which requires the model to answer questions related to a video. This task is challenging as video contents are complex with strong irrelevant backgrounds. Besides, most QA pairs in video QA task are related to more than one action with temporal cues. To answer the question correctly, the model is required not 

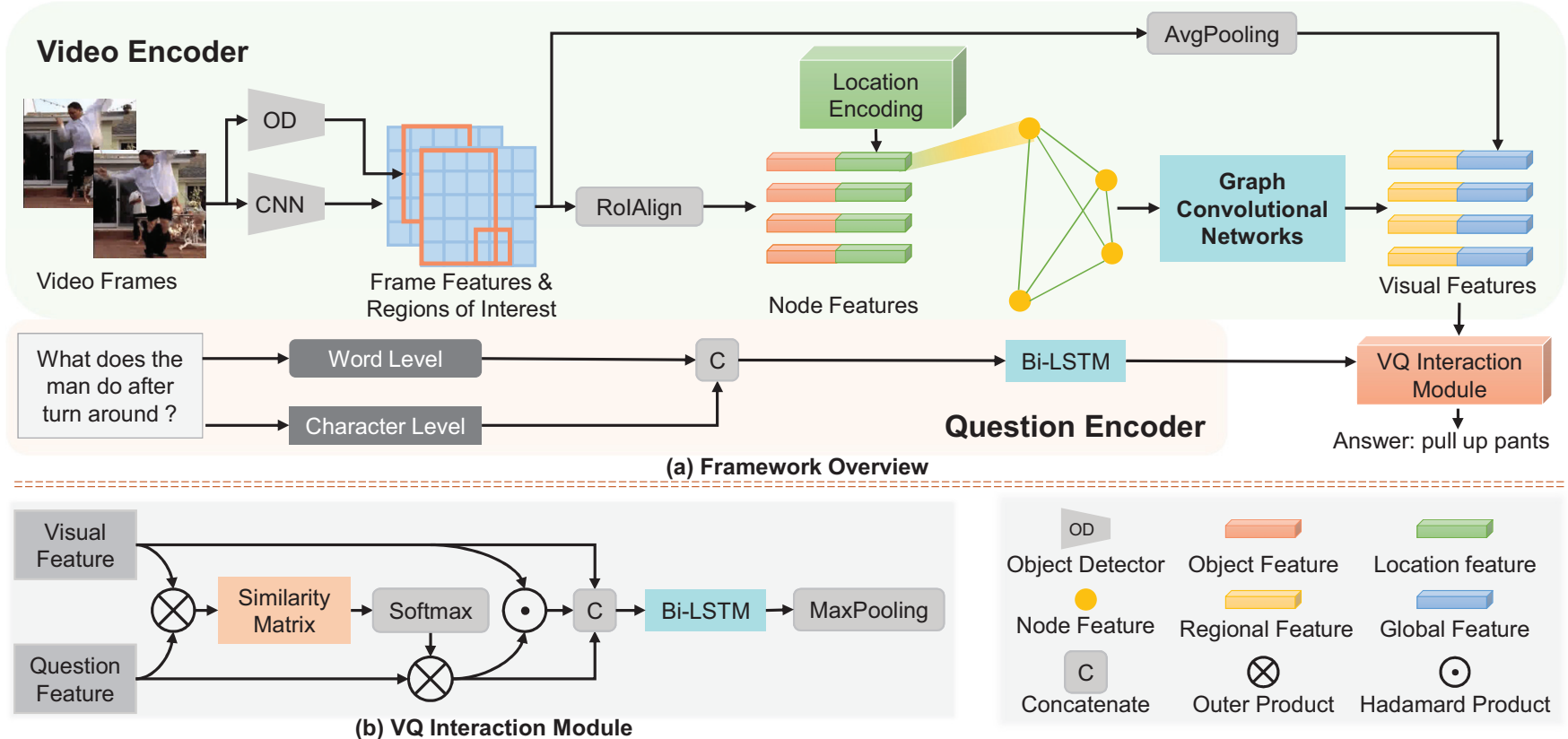

Figure 2: Illustration of the proposed method. L-GCN consists of two streams, namely the question encoder stream and the video encoder stream, which process queries and video contents, respectively. The outputs of two streams are combined with a visual-question (VQ) interaction module. The location-aware graph built on objects considers both interactions of objects and their temporal location information.

only to recognize the actions correctly from complex contents but also to be aware of their temporal order.

\subsection{General Scheme}

The general scheme of our method is shown in Figure 2, which consists of two streams. The first stream is regarding a question encoder, which processes queries with a BiLSTM. The second stream is related to a video encoder, which focuses on understanding video contents by exploiting a location-aware graph built on objects. The outputs of two streams are then combined by a visual-question (VQ) interaction module, which employs an attention mechanism to explore which question words are more relevant to the visual representation. Last, the answer is predicted by applying an FC layer on top of the VQ interaction module.

In this paper, the location-aware graph plays a critical role. Specifically, we use an object graph $\mathcal{G}=(\mathcal{V}, \mathcal{E})$ to model the relationships between objects in a video. Note the temporal ordering of actions in the video is important for answer reasoning w.r.t. a question in a video QA task. We thus propose to integrate the spatial and temporal location information into the object features of each node in a graph (See details in Section 3.4). In this way, we can exploit both spatial and temporal order information of actions for temporally related answer reasoning.

For convenience, we present the overall training process in Algorithm 1. In the following, we first describe the question encoder. Then we depict the construction of the location-aware graph and the graph convolution for message passing, followed by description of visual encoder. Af-

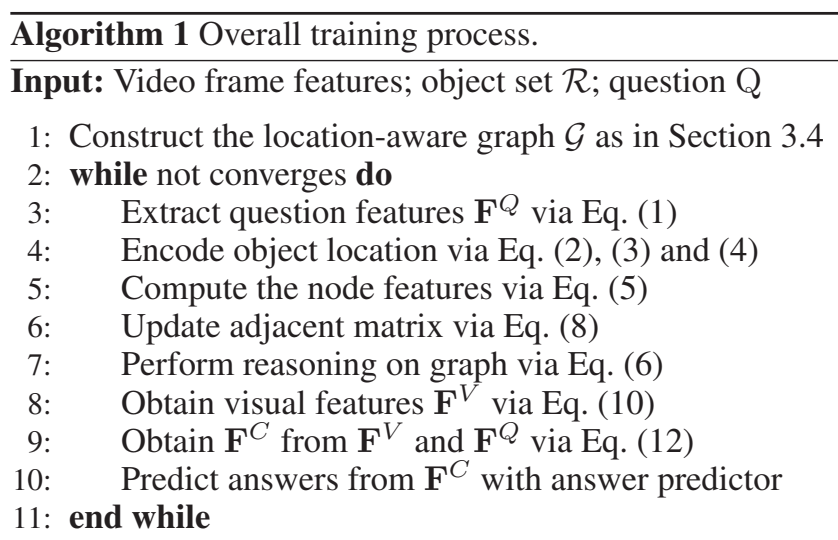

Output: Trained model for video QA

ter that, we detail the visual-question interaction module. Last, we present the answer reasoning and loss functions.

\subsection{Question Encoder Stream}

Given a question sentence, the question encoder is to model the question for video QA. To handle the out-of-vocabulary words as well as the misspelling words, we apply both character embedding $\mathbf{Q}^{c} \in \mathbb{R}^{\kappa \times c \times d_{c}}$ and word embedding $\mathbf{Q}^{w} \in \mathbb{R}^{\kappa \times d_{w}}$ to represent a question $\mathrm{Q}$ with $\kappa$ words, where $d_{c}$ and $d_{w}$ denote the dimensions of character embedding and word embedding, respectively.

In the optimization, the word embedding function is ini- 
tialized with a pre-trained 300-dimension GloVe (Pennington, Socher, and Manning 2014), and the character embedding function is randomly initialized. Given the character and word embeddings, the question embedding can be represented by a two-layer highway network $h(\cdot, \cdot)$ (Srivastava, Greff, and Schmidhuber 2015), which is proven to be effective to solve the training difficulties, that is:

$$
\mathbf{Q}=h\left(\mathbf{Q}^{w}, g\left(\mathbf{Q}^{c}\right)\right)
$$

where the character embedding is further processed by a $g(\cdot)$ which consists of a 2D convolutional layer.

To better encode the question, we feed the question embedding $\mathrm{Q}$ into a bi-directional LSTM (Bi-LSTM). Then we obtain the question feature $\mathbf{F}^{Q}$ by stacking the hidden states of the Bi-LSTM from both directions at each time step.

\subsection{Location-aware Graph Construction}

Given a video with $K$ detected objects for each frame, we seek to represent the video into a graph. Noting that actions can be inferred from the interaction between objects, we thus construct a fully-connected graph on the detected objects. We may use object features to represent each node. However, this node type ignores the location information of objects, which is vital for temporally related answer reasoning. To address this, we will describe how to encode the location information with so-called location features. With location features, we are able to construct a location-aware graph, namely, we concatenate both object appearance and location features as node features.

Location Encoding. Given a detected object in the $n^{t h}$ frame with spatial location $\mathbf{b}$ and aligned feature $\mathbf{o}$, we encode its spatial location feature $\mathbf{d}^{s}$ with a Multilayer Perceptron $(\mathrm{MLP}(\cdot))$ which consists of two FC layers and a ReLU activation function (Nair and Hinton 2010), that is:

$$
\mathbf{d}^{s}=\operatorname{MLP}(\mathbf{b})
$$

where $\mathbf{b}$ is represented by the top-left coordinate and the width and the height of detected objects.

Moreover, we also encoder temporal location feature $\mathbf{d}^{t}$ of objects using sine and cosine functions of different frequencies (Vaswani et al. 2017) as follows:

$$
\begin{gathered}
d_{2 j}^{t}=\sin \left(n / 10000^{2 j / d_{p}}\right), \\
d_{2 j+1}^{t}=\cos \left(n / 10000^{2 j / d_{p}}\right),
\end{gathered}
$$

where $d_{i}^{t}$ is the $i$-th entry of the temporal location feature $\mathbf{d}^{t}$, and $d_{p}$ is its dimension. Then, the feature of each graph node can be defined as:

$$
\mathbf{v}=\left[\mathbf{o} ; \mathbf{d}^{s} ; \mathbf{d}^{t}\right]
$$

where $[\cdot ; \cdot ; \cdot]$ concatenates three vectors into a longer vector. In this way, each node in the graph contains not only the object appearance features but also the location information.

\subsection{Reasoning with Graph Convolution}

Given the constructed location-aware graph, we perform graph convolution to obtain the regional features. In our implementation, we build $P$-layer graph convolutions. Specifically, for the $p$-th layer $(1 \leq p \leq P)$, the graph convolution can be formally represented as:

$$
\mathbf{X}^{(p)}=\mathbf{A}^{(p)} \mathbf{X}^{(p-1)} \mathbf{W}^{(p)},
$$

where $\mathbf{X}^{(p)}$ is the hidden features of the $p$-th layer; $\mathbf{X}^{(0)}$ is the input node features $\mathbf{v}$ in Eq. $(5) ; \mathbf{A}^{(p)}$ is the adjacency matrix calculated from the node features in the $p$-th layer; and $\mathbf{W}^{(p)}$ is the trainable weight matrix. Let $\mathbf{X}^{(P)}$ be the output of the last layer of the $P$-layer GCNs. Then, we define the regional features $\mathbf{F}^{R}$ as:

$$
\mathbf{F}^{R}=\mathbf{X}^{(P)}+\mathbf{X}^{(0)} .
$$

This can be considered as a skip connection of input $\mathbf{X}^{(0)}$ and output $\mathbf{X}^{(P)}$, and it helps to improve the training performance, similar to ResNet (He et al. 2016). In our method, the adjacency matrix is a learnable matrix, which is able to simultaneously infer a graph by learning the weight of all edges. We calculate the adjacency matrix by:

$$
\mathbf{A}^{(p)}=\operatorname{softmax}\left(\mathbf{X}^{(p-1)} \mathbf{W}_{1} \cdot\left(\mathbf{X}^{(p-1)} \mathbf{W}_{2}\right)^{\top}\right),
$$

where $\mathbf{W}_{1}$ and $\mathbf{W}_{2}$ are projection matrices. The softmax operation is performed in the row axis.

\subsection{Visual Encoder Stream}

The visual encoder is to model video contents via object interaction for video QA. Given a $N$-frame video, we extract frame features using a fixed feature extractor (e.g., ResNet152). At the same time, $K$ bounding boxes are detected for each frame by an off-the-shelf object detector. The object features o are obtained using RoIAlign (He et al. 2017) on top of the image features, followed by an FC layer and ELU activation function (Clevert, Unterthiner, and Hochreiter 2016) to reduce dimension.

Given the detected object set $\mathcal{R}$, we construct a locationaware graph $\mathcal{G}(\mathcal{V}, \mathcal{E})$ on the objects. Then, we perform graph convolution to enable the message passing between objects through edges, which can be formally represented as:

$$
\operatorname{GCN}\left(\mathcal{G}(\mathcal{V}, \mathcal{E}),\left\{\left[\mathbf{o}_{t} ; f\left(\mathbf{b}_{t}\right)\right]\right\}_{t=1}^{T}\right),
$$

where $[\cdot ; \cdot]$ indicates the concatenation of vectors and $f(\cdot)$ denotes for any mapping function, e.g., multi-layer perceptron (MLP). The output of GCNs is termed as regional features $\mathbf{F}^{R}$. Besides, in order to introduce the context information, we apply global average pooling on the frame features to generate global features $\mathbf{F}^{G}$.

The global features are further processed by a 1D convolutional layer and an ELU activation function to merge the information from neighbor frames. After that, we replicate the global features $K$ times and employ Multilayer Perceptron (MLP) (with one hidden layer and an ELU activation function) to merge the concatenation of $\mathbf{F}^{R}$ and $\mathbf{F}^{G}$, which yields visual features $\mathbf{F}^{V}$ and that is:

$$
\mathbf{F}^{V}=\operatorname{MLP}\left(\left[\mathbf{F}^{R}, \mathbf{F}^{G}\right]\right) .
$$




\subsection{Visual-question Interaction Module}

After obtaining visual and question representations, we propose a visual-question (VQ) interaction module to combine them for predicting answer. The framework of VQ interaction module is shown in Figure 2(b). We first map $\mathbf{F}^{V}$ and $\mathbf{F}^{Q}$ into the same subspace with dimension $d_{s}$ through two independent FC layers, leading to $\mathbf{F}^{V} \in \mathbb{R}^{T \times d_{s}}$ and $\mathbf{F}^{Q} \in \mathbb{R}^{L \times d_{s}}$. Then, we explore which question words are more relevant to each visual representation for video QA. In this paper, we leverage attention mechanism to learn a cross modality representation inspired by (Seo et al. 2017).

Specifically, we first calculate similarity matrix $\mathbf{S}$ between $\mathbf{F}^{V}$ and $\mathbf{F}^{Q}$ via dot product together with a softmax function applying along each row, that is:

$$
\mathbf{S}=\operatorname{softmax}\left(\mathbf{F}^{V}\left(\mathbf{F}^{Q}\right)^{\top}\right) .
$$

Then, we calculate the weighted question features $\widetilde{\mathbf{F}}^{Q}$ corresponding to each visual feature via dot product between $\mathbf{S}$ and $\mathbf{F}^{Q}$. The cross modality representation $\mathbf{F}^{C} \in \mathbb{R}^{P \times 3 d_{s}}$ is calculated by:

$$
\mathbf{F}^{C}=\left[\mathbf{F}^{V}, \widetilde{\mathbf{F}}^{Q}, \mathbf{F}^{V} \odot \widetilde{\mathbf{F}}^{Q}\right]
$$

where $\odot$ means the element-wise product operation. To yield the final representation for answer prediction, we leverage a Bi-LSTM followed by a max pooling layer across the dimension $T$.

\subsection{Answer Reasoning and Loss Function}

The questions for video QA can be summarized as three types: multiple-choice, open-ended and counting. In this subsection, we will describe how to predict answers for each question type given cross modality features $\mathbf{F}^{C}$.

Multiple-choice question: for this kind of questions, there exist $U$ choices and the model is required to choose the correct one. We first embed the content of each choice in the same way as question encoding described in Section 3.3, leading to $U$ independent answer features $\mathbf{F}^{A}$. Then, each answer feature is interacted with visual features in the way described in Section 3.7, where we replace the question feature by answer question, yielding the weighted answer features $\widetilde{\mathbf{F}}^{A}$. Then, the cross modality representation $\mathbf{F}^{C}$ in Eq. (12) is constructed as $\left[\mathbf{F}^{V}, \widetilde{\mathbf{F}}^{Q}, \widetilde{\mathbf{F}}^{A}, \mathbf{F}^{V} \odot \widetilde{\mathbf{F}}^{Q}, \mathbf{F}^{V} \odot \widetilde{\mathbf{F}}^{A}\right]$. We leverage an identical FC layer on $U$ cross modality representations to predict scores $\mathcal{A}=\left\{a_{1}, \ldots, a_{U}\right\}$. The scores are processed by a softmax function. We use cross entropy loss as the loss function:

$$
L_{M}=-\sum_{i=1}^{U} y_{i} \log \left(\frac{e^{a_{i}}}{\sum_{j=1}^{U} e^{a_{j}}}\right)
$$

where $y_{i}=1$ if answer $a_{i}$ is the right choice, otherwise $y_{i}=0$. We take the choice with the highest score as the prediction.

Open-ended question: for these questions, the model is required to choose a correct word as answer from the predefined answer set of $C$ candidate words in total. We predict the scores $\mathcal{A}=\left\{a_{1}, \ldots, a_{C}\right\}$ of each candidate word using an FC layer together with a softmax layer. Also, we use the cross entropy loss as the loss function:

$$
L_{O}=-\sum_{i=1}^{C} y_{i} \log \left(\frac{e^{a_{i}}}{\sum_{j=1}^{C} e^{a_{j}}}\right),
$$

where $y_{i}=1$ if answer $a_{i}$ is the right answer, otherwise $y_{i}=$ 0 . We take the word with the highest score as our prediction.

Counting question: for these questions, the model is required to predict a number ranging from 0 to 10 . We leverage an $\mathrm{FC}$ layer upon $\mathbf{F}^{C}$ to predict the number. We use mean square error loss to train the model:

$$
L_{C}=\|\mathbf{x}-\mathbf{y}\|_{2}^{2}
$$

where $\mathbf{x}$ is the predicted number, $\mathbf{y}$ is the ground truth. During the testing, the prediction is rounded to the nearest integer and clipped within 0 to 10 .

\section{Experiments}

In this section, we first introduce three benchmark datasets and implementation details. Then, we compare the performance of our model with the state-of-the-art methods. Last, we perform ablation studies to understand the effect of each component.

\subsection{Datasets}

We evaluate our method on three video QA datasets. The statistics of the datasets are listed in Table 1. More details are given below.

TGIF-QA (Jang et al. 2017) consists of 165K QA pairs from $72 \mathrm{~K}$ animated GIFs. The QA-pairs are splited into four tasks: 1) Action: a multiple-choice question recognizing action repeated certain times; 2) Transition (Trans.): a multiple-choice question asking about the state transition; 3) FrameQA: an open-ended question that can be inferred from one frame in videos; 4) Count: an open-ended question counting the number of repetition of an action. The multiplechoice questions in this dataset have five options and the open-ended questions are with a pre-defined answer set of size 1,746 .

Youtube2Text-QA (Ye et al. 2017) includes the videos from MSVD video set (Chen and Dolan 2011) and the question-answer pairs collected from Youtube2Text (Guadarrama et al. 2013) video description corpus. It consists of open-ended and multiple-choice questions, which are divided into three types (i.e., what, who and others).

MSVD-QA (Xu et al. 2017) is based on MSVD video set. It consists of five types of questions, including what, who, how, when and where. All questions are open-ended with a pre-defined answer set of size 1,000.

\subsection{Implementation Details}

Evaluation metrics. (1) For the "Count" task in TGIF-QA dataset, we adopt the Mean Square Error (MSE) between the predicted answer and the ground truth answer as the evaluation metric. (2) For all other tasks in our experiments, we use accuracy to evaluate the performance. 
Table 1: Statistics of three video QA datasets. \#MC denotes the number of options for multiple-choice questions.

\begin{tabular}{c|ccccccc}
\hline Dataset & Vocab. size & \#Video & \#Question & Answer size & \#MC & Feature type & \#Sampled frame \\
\hline TGIF-QA & 8,000 & 71,741 & 165,165 & 1,746 & 5 & ResNet-152 & 35 \\
Youtube2Text-QA & 6,500 & 1,970 & 99,429 & 1,000 & 4 & ResNet-101+C3D & 40 \\
MSVD-QA & 4,000 & 1,970 & 50,505 & 1,000 & NA & VGG+C3D & 20 \\
\hline
\end{tabular}

Table 2: Comparisons with state-of-the-arts on TGIF-QA dataset. R, C and F denote features extracted by ResNet, C3D and Optical Flow, respectively.

\begin{tabular}{c|cccc}
\hline Model & Action & Trans. & FrameQA & Count (MSE) \\
\hline ST-VQA(R+C) & 60.8 & 67.1 & 49.3 & 4.28 \\
Co-Mem(R+F) & 68.2 & 74.3 & 51.5 & 4.10 \\
PSAC(R) & 70.4 & 76.9 & 55.7 & 4.27 \\
HME(R+C) & 73.9 & 77.8 & 53.8 & 4.02 \\
\hline Ours(R) & $\mathbf{7 4 . 3}$ & $\mathbf{8 1 . 1}$ & $\mathbf{5 6 . 3}$ & $\mathbf{3 . 9 5}$ \\
\hline
\end{tabular}

Training details. We convert all the words in the question and answer to lower cases, and then transform each word to a 300-dimension vector with a pre-trained GloVe model (Pennington, Socher, and Manning 2014). For fair comparisons, we adopt the same feature extractors as those are used in the compared methods. More details can be found in Table 1. We use Mask R-CNN (He et al. 2017) as object detector and select $K$ detected objects with the highest score for each frame. By default, $K$ is set to 5 . The number of GCNs layers is set to 2. We employ a Adam optimizer (Kingma and $\mathrm{Ba} 2015$ ) to train the network with an initial learning rate of $1 \mathrm{e}-4$. We set the batch size to 64 and 128 for multiple-choice and open-ended tasks, respectively.

\subsection{Comparison with State-of-the-art Results}

Results on TGIF-QA. We compare our L-GCN with the state-of-the-art methods, including ST-VQA (Jang et al. 2017), Co-Men (Gao et al. 2018), PSAC (Li et al. 2019b) and HME (Chenyou Fan 2019). From Table 2, our L-GCN achieves the best performance on four tasks. It is worth noting that our method outperforms HME, ST-VQA and CoMem by a large margin even if they use additional features (i.e., C3D features (Tran et al. 2015) and optical flow feature) to model actions. These results demonstrate the effectiveness of leveraging an object graph to capture the objectobject interaction and perform reasoning.

Results on Youtube2Text-QA. For further comparison, we test our model on a more challenging dataset Youtube2Text-QA. This dataset consists of open-ended and multiple-choice questions, which are divided into three categories (i.e., what, who and others). We consider two state-ofthe-art baseline methods (HME and r-ANL (Ye et al. 2017)), and report the results in Table 3.

From Table 3, compared with the baselines, our method achieves better performance in overall accuracy in both multi-choice and open-ended questions. More specifically, for multiple-choice questions, we achieve the best performance on what and who tasks. The relatively poor performance on others task cannot represent the ability of different
Table 3: Comparisons with state-of-the-art methods on Youtube2Text-QA.

\begin{tabular}{c|c|ccc|c}
\hline Task & Method & What & Who & Other & All \\
\hline \multirow{3}{*}{ Multiple-Choice } & r-ANL & 63.3 & 36.4 & 84.5 & 52.0 \\
& HME & 83.1 & 77.8 & $\mathbf{8 6 . 6}$ & 80.8 \\
& Ours & $\mathbf{8 6 . 0}$ & $\mathbf{8 1 . 5}$ & 80.6 & $\mathbf{8 3 . 9}$ \\
\hline \multirow{3}{*}{ Open-Ended } & r-ANL & 21.6 & 29.4 & $\mathbf{8 0 . 4}$ & 26.2 \\
& HME & $\mathbf{2 9 . 2}$ & 28.7 & 77.3 & 30.1 \\
& Ours & 24.5 & $\mathbf{5 3 . 2}$ & 70.4 & $\mathbf{3 8 . 0}$ \\
\hline
\end{tabular}

Table 4: Comparisons with state-of-the-arts on MSVD-QA.

\begin{tabular}{c|cccc|c}
\hline Model & ST-VQA & Co-Mem & AMU & HME & Ours \\
\hline Acc & 31.3 & 31.7 & 32.0 & 33.7 & $\mathbf{3 4 . 3}$ \\
\hline
\end{tabular}

models because this kind of questions only occupies $2 \%$ of all QA pairs. For open-ended questions, our L-GCN significantly improves the accuracy from $29.4 \%$ to $53.2 \%$ on who task, where most questions are related to the subject of actions. This demonstrates the superiority of leveraging object features, which explicitly localizes the object for video QA task.

Results on MSVD-QA. In Table 4, we compare our LGCN with ST-VQA, Co-Mem, AMU (Xu et al. 2017) and HME on MSVD-QA dataset. From Table 4, our L-GCN achieves the most promising performance in overall accuracy, which demonstrates the superiority of the proposed method on the non-trivial scenarios.

\subsection{Ablation Study}

Impact of each component. We first construct a simple variant of the proposed method as baseline, which uses only the global frame features $\mathbf{F}^{G}$ to generate visual features $\mathbf{F}^{V}$ via Eq. (10). Then, the object features, GCNs, and location features will be incorporated into the baseline progressively to generate visual features in higher quality, and we denote them as "OF", "GCNs" and "Loc", respectively. "FC"'and "LSTM" represent the models where GCNs are replaced by two Fully-Connected (FC) layers or a 2-layer LSTM, respectively. "Loc_T"'and "Loc_S" represent the location features which only consist of temporal or spatial location information, respectively.

We show the results on TGIF-QA dataset in Table 5. (1) Compared with the baseline, incorporating object features boosts the performance in all tasks consistently, demonstrating the effectiveness of using detected objects for video QA task. We speculate that the detected objects explicitly help the model exclude irrelevant background. (2) Applying GCNs on object features further boosts the performance, 
Table 5: Performance comparisons of different variants on TGIF-QA. "OF" and "Loc" denote object and location features, respectively.

\begin{tabular}{l|cccc}
\hline Model & Action & Trans. & FrameQA & Count \\
\hline baseline & 70.58 & 79.59 & 55.37 & 4.33 \\
baseline+OF & 72.82 & 80.10 & 55.79 & 4.24 \\
baseline+OF+GCNs & 74.10 & 80.39 & 56.10 & 4.15 \\
baseline+OF+GCNs+Loc & $\mathbf{7 4 . 3 2}$ & $\mathbf{8 1 . 1 3}$ & $\mathbf{5 6 . 3 2}$ & $\mathbf{3 . 9 5}$ \\
\hline baseline+OF+FC & 72.96 & 80.18 & 55.94 & 4.22 \\
baseline+OF+LSTM & 72.65 & 80.07 & 55.49 & 4.25 \\
baseline+OF+GCNs+Loc_T & 73.75 & 80.97 & 55.54 & 4.17 \\
baseline+OF+GCNs+Loc_S & 73.58 & 80.89 & 56.07 & 4.12 \\
\hline
\end{tabular}

Table 6: Ablation study on \#GCNs layers on TGIF-QA.

\begin{tabular}{c|cccc}
\hline \#GCNs layers & Action & Trans. & FrameQA & Count \\
\hline 1 & 74.24 & 81.02 & 55.97 & 4.16 \\
2 & $\mathbf{7 4 . 3 2}$ & 81.13 & $\mathbf{5 6 . 3 2}$ & $\mathbf{3 . 9 5}$ \\
3 & $\mathbf{7 4 . 3 2}$ & $\mathbf{8 1 . 5 8}$ & 56.23 & 4.16 \\
4 & 73.97 & 80.86 & 56.01 & 4.10 \\
\hline
\end{tabular}

demonstrating the importance of modeling relationships between objects through GCNs. On the other hand, using FC layer or LSTM only brings minor increases or even drops the performance. This is not surprising because the model cannot learn object-object relationship when applying FC layer on each object separately. Besides, objects in different spatial locations cannot be regarded as a sequence and thus LSTM is not suitable for modeling their relationship. (3) Adding location features further increases the performance. Especially, the improvements on the task of transition and count are more significant. One possible reason is that these two tasks are more sensitive to the knowledge of event's order, where the transition task asks about the action transition and the count task asks the number of repetition of an action. We also try to only incorporate temporal or spatial location information into L-GCN. The performance decreases compared to the variant using both location types, demonstrating that these two location information are complementary and both vital for video QA task.

Impact of \#GCNs layers and detected objects. In this paper, we propose to leverage GCNs on detected objects to learn actions. Here, we conduct ablation studies on the depth of GCNs and the number of the objects in each frame. From Table 6, GCNs with two layers performs best on three tasks. Considering the efficiency and performance, we leverage 2layer GCNs by default. Besides, as shown in Table 7, GCNs with 5 detected objects achieves the best performance on three tasks. It is not surprising that the network with 2 detected objects performs worst because the network may neglect some important objects. Additionally, as most of the question answering pairs in TGIF-QA dataset are only relative to a few salient objects, feeding too many objects into network may cripple the performance. By default, we leverage 5 detected objects in experiments.

\subsection{Qualitative Analysis}

We demonstrate the similarity matrix in the GCNs using two examples in Figure 3. We draw two conclusions from these
Table 7: Performance comparisons between different numbers of detected objects on TGIF-QA dataset.

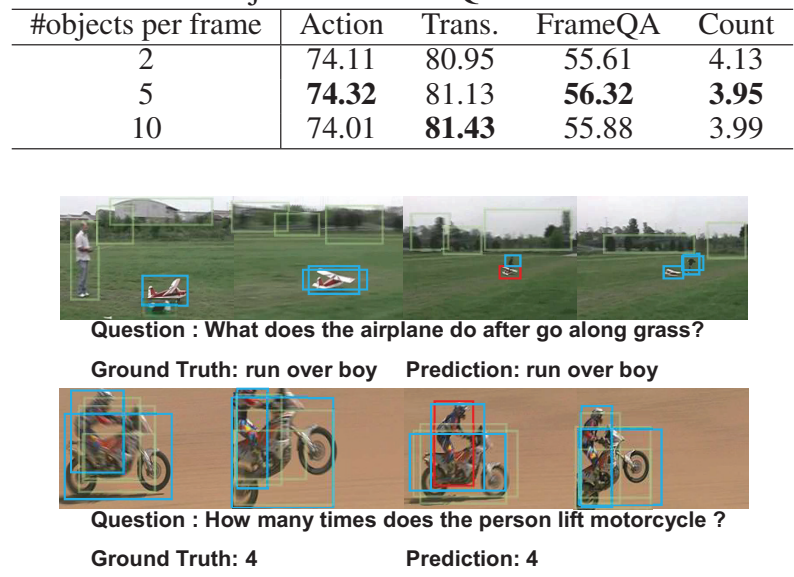

Figure 3: Visualization on TGIF-QA dataset. The boxes in transparent green are $K$ detected objects. The boxes in red are the query object. The boxes in blue are the objects with high values regarding to query object in the adjacent matrix.

examples: 1) Almost all salient objects which are related to question answering pair have been detected beforehand, such as the airplane and the boy in example 1, the man and the motorcycle in example 2, etc. These detected objects explicitly help the network avoid the influence from complex irrelevant background content. 2) Our graph not only captures relationships between similar objects in different frames but also focuses on semantic similarity. For the first example, the airplane is correlative to not only itself in different frames but also the little boy. This is helpful to recognize the action of "airplane running over boy".

\section{Conclusion}

In this paper, we have proposed a location-aware graph to model the relationships between detected objects for video QA task. Compared with existing spatio-temporal attention mechanism, L-GCN is able to explicitly get rid of the influences from irrelevant background content. Moreover, our network is aware of the spatial and temporal location of events, which is important for predicting correct answer. Our method outperforms state-of-the-art techniques on three benchmark datasets.

\section{Acknowledgment}

This work was partially supported by Guangdong Provincial Scientific and Technological Funds under Grants 2018B010107001, National Natural Science Foundation of China (NSFC) 61602185, key project of NSFC (No. 61836003), Program for Guangdong Introducing Innovative and Entrepreneurial Teams 2017ZT07X183, Tencent AI Lab Rhino-Bird Focused Research Program (No. JR201902), Natural Science Foundation of Guangdong Province under Grant 2016A030310423, Fundamental Research Funds for the Central Universities D2191240. 


\section{References}

Anderson, P.; He, X.; Buehler, C.; Teney, D.; Johnson, M.; Gould, S.; and Zhang, L. 2018. Bottom-up and top-down attention for image captioning and visual question answering. In $C V P R$.

Cadène, R.; Ben-younes, H.; Cord, M.; and Thome, N. 2019. MUREL: multimodal relational reasoning for visual question answering. In $C V P R$.

Cao, J.; Guo, Y.; Wu, Q.; Shen, C.; and Tan, M. 2018. Adversarial learning with local coordinate coding. In ICML.

Cao, J.; Mo, L.; Zhang, Y.; Jia, K.; Shen, C.; and Tan, M. 2019. Multi-marginal wasserstein gan. In NeurIPS.

Chen, D. L., and Dolan, W. B. 2011. Collecting highly parallel data for paraphrase evaluation. In $A C L$.

Chenyou Fan, Xiaofan Zhang, S. Z. W. W. C. Z. H. H. 2019. Heterogeneous memory enhanced multimodal attention model for video question answering. In $C V P R$.

Clevert, D.; Unterthiner, T.; and Hochreiter, S. 2016. Fast and accurate deep network learning by exponential linear units (elus). In $I C L R$.

Gan, C.; Wang, N.; Yang, Y.; Yeung, D.-Y.; and Hauptmann, A. G. 2015. Devnet: A deep event network for multimedia event detection and evidence recounting. In $C V P R$.

Gan, C.; Li, Y.; Li, H.; Sun, C.; and Gong, B. 2017. VQS: Linking segmentations to questions and answers for supervised attention in vqa and question-focused semantic segmentation. In ICCV.

Gan, C.; Zhao, H.; Chen, P.; Cox, D.; and Torralba, A. 2019. Selfsupervised moving vehicle tracking with stereo sound. In ICCV.

Gao, J.; Ge, R.; Chen, K.; and Nevatia, R. 2018. Motionappearance co-memory networks for video question answering. In CVPR.

Goyal, Y.; Khot, T.; Summers-Stay, D.; Batra, D.; and Parikh, D. 2017. Making the V in VQA matter: Elevating the role of image understanding in visual question answering. In $C V P R$.

Guadarrama, S.; Krishnamoorthy, N.; Malkarnenkar, G.; Venugopalan, S.; Mooney, R. J.; Darrell, T.; and Saenko, K. 2013. Youtube2text: Recognizing and describing arbitrary activities using semantic hierarchies and zero-shot recognition. In ICCV.

Guo, Y.; Chen, Q.; Chen, J.; Wu, Q.; Shi, Q.; and Tan, M. 2019a. Auto-embedding generative adversarial networks for high resolution image synthesis. TMM.

Guo, Y.; Zheng, Y.; Tan, M.; Chen, Q.; Chen, J.; Zhao, P.; and Huang, J. 2019b. NAT: Neural architecture transformer for accurate and compact architectures. In NeurIPS.

He, K.; Zhang, X.; Ren, S.; and Sun, J. 2016. Deep residual learning for image recognition. In $C V P R$.

He, K.; Gkioxari, G.; Dollár, P.; and Girshick, R. B. 2017. Mask R-CNN. In ICCV.

Jang, Y.; Song, Y.; Yu, Y.; Kim, Y.; and Kim, G. 2017. TGIF-QA: toward spatio-temporal reasoning in visual question answering. In CVPR.

Kingma, D. P., and Ba, J. 2015. Adam: A method for stochastic optimization. In ICLR.

Kipf, T. N., and Welling, M. 2017. Semi-supervised classification with graph convolutional networks. In ICLR.

Lei, J.; Yu, L.; Bansal, M.; and Berg, T. L. 2018. TVQA: localized, compositional video question answering. In EMNLP.

Li, L.; Gan, Z.; Cheng, Y.; and Liu, J. 2019a. Relation-aware graph attention network for visual question answering. In ICCV.
Li, X.; Song, J.; Gao, L.; Liu, X.; Huang, W.; He, X.; and Gan, C. 2019b. Beyond rnns: Positional self-attention with co-attention for video question answering. In $A A A I$.

Long, X.; Gan, C.; De Melo, G.; Wu, J.; Liu, X.; and Wen, S. 2018. Attention clusters: Purely attention based local feature integration for video classification. In CVPR.

Nair, V., and Hinton, G. E. 2010. Rectified linear units improve restricted boltzmann machines. In ICML.

Nguyen, D., and Okatani, T. 2018. Improved fusion of visual and language representations by dense symmetric co-attention for visual question answering. In $C V P R$.

Norcliffe-Brown, W.; Vafeias, S.; and Parisot, S. 2018. Learning conditioned graph structures for interpretable visual question answering. In NeurIPS.

Pennington, J.; Socher, R.; and Manning, C. D. 2014. Glove: Global vectors for word representation. In EMNLP.

Seo, M. J.; Kembhavi, A.; Farhadi, A.; and Hajishirzi, H. 2017. Bidirectional attention flow for machine comprehension. In ICLR. Singh, A.; Natarajan, V.; Jiang, Y.; Chen, X.; Shah, M.; Rohrbach, M.; Batra, D.; and Parikh, D. 2018. Pythia-a platform for vision \& language research. In SysML Workshop, NeurIPS.

Srivastava, R. K.; Greff, K.; and Schmidhuber, J. 2015. Highway networks. Arxiv abs/1505.00387.

Tan, M.; Shi, Q.; van den Hengel, A.; Shen, C.; Gao, J.; Hu, F.; and Zhang, Z. 2015. Learning graph structure for multi-label image classification via clique generation. In $C V P R$.

Tan, M.; Tsang, I. W.; and Wang, L. 2014. Towards ultrahigh dimensional feature selection for big data. Journal of Machine Learning Research 15.

Tran, D.; Bourdev, L. D.; Fergus, R.; Torresani, L.; and Paluri, M. 2015. Learning spatiotemporal features with 3 d convolutional networks. In $I C C V$.

Vaswani, A.; Shazeer, N.; Parmar, N.; Uszkoreit, J.; Jones, L.; Gomez, A. N.; Kaiser, L.; and Polosukhin, I. 2017. Attention is all you need. In NeurIPS.

Wang, X., and Gupta, A. 2018. Videos as space-time region graphs. In $E C C V$.

Wu, W.; He, D.; Tan, X.; Chen, S.; and Wen, S. 2019. Multiagent reinforcement learning based frame sampling for effective untrimmed video recognition. In ICCV.

Xiong, C.; Merity, S.; and Socher, R. 2016. Dynamic memory networks for visual and textual question answering. In ICML.

Xu, D.; Zhao, Z.; Xiao, J.; Wu, F.; Zhang, H.; He, X.; and Zhuang, Y. 2017. Video question answering via gradually refined attention over appearance and motion. In ACMMM.

Yang, Z.; He, X.; Gao, J.; Deng, L.; and Smola, A. J. 2016. Stacked attention networks for image question answering. In CVPR.

Ye, Y.; Zhao, Z.; Li, Y.; Chen, L.; Xiao, J.; and Zhuang, Y. 2017. Video question answering via attribute-augmented attention network learning. In SIGIR.

Yi, K.; Gan, C.; Li, Y.; Kohli, P.; Wu, J.; Torralba, A.; and Tenenbaum, J. B. 2019. Clevrer: Collision events for video representation and reasoning. arXiv preprint arXiv: 1910.01442.

Zeng, R.; Gan, C.; Chen, P.; Huang, W.; Wu, Q.; and Tan, M. 2019a. Breaking winner-takes-all: Iterative-winners-out networks for weakly supervised temporal action localization. IEEE Transactions on Image Processing 28(12).

Zeng, R.; Huang, W.; Tan, M.; Rong, Y.; Zhao, P.; Huang, J.; and Gan, C. 2019b. Graph convolutional networks for temporal action localization. In $I C C V$. 